\title{
Los alcances en la producción agrícola chiapaneca. Una reflexión sobre la soberanía alimentaria en la región
}

\author{
The Scopes in Chiapas Agricultural Production. \\ A Region's Reflection on Food Sovereignty
}

\author{
María de Lourdes Flores* (iD https://orcid.org/0000-0002-2997-2001
}

\section{Resumen}

El objetivo del artículo es mostrar las condiciones globales y sociales en las cuales se enmarca la producción agrícola en el campo chiapaneco dentro del contexto de los programas de reconversión agrícola y su pertinencia en el marco de la soberanía alimentaria. Mediante una revisión de fuentes secundarias e información derivada del Servicio de Información Agroalimentaria y Pesquera entre 2003 y 2016, se muestran datos descriptivos y comparativos respecto a la producción de cultivos básicos y estratégicos. Se discute la tendencia a llevar la producción a niveles competitivos en afán de integrarse a lógicas globales que poco reflejan la realidad y las necesidades de los productores chiapanecos de las regiones la Frailesca, Metropolitana, Soconusco y Meseta Comiteca. Se concluye que las políticas públicas no han sido adecuadas para las condiciones locales; por lo tanto, no pueden generar el impulso económico ni tienen miras al desarrollo social de estas regiones de Chiapas. Se recomienda el impulso a la soberanía alimentaria como vía de salida de la pobreza, la desigualdad y la merma de alimentos.

Palabras clave: reconversión agrícola; rotación de cultivos; soberanía alimentaria; producción de alimentos; política agrícola; sostenibilidad económica; desarrollo socioeconómico.

\begin{abstract}
The purpose of this paper is to show global and social conditions that frame Chiapas' agricultural production within the agricultural re-conversion programs context and their appropriateness within food sovereignty. Revision of secondary resources and of Agrifood and Fisheries Information Service's knowledge between 2003 and 2016 led to descriptive and comparative data about staple and strategic crops production. There is a tendency to point the way of production to a competitive level in order to incorporate it to global logic, which poorly reflect Chiapas producers' reality and needs, especially from Frailesca, Metropolitana, Soconusco, and Meseta Comiteca. Public policies have not been suitable for local conditions hence cannot generate economic impulse or social development in these Chiapas' regions. Recommendations concentrate on give impulse to food sovereignty as an exit rout to poverty, inequality and decline in food.

Keywords: agricultural reconversion; crop rotation; food sovereignty; food production; agricultural policy; economic sustainability; socio-economic development.
\end{abstract}

Cómo citar: Flores, M.L. (2019). Los alcances en la producción agrícola chiapaneca. Una reflexión sobre la soberanía alimentaria en la región. región y sociedad, 3 I, el I77. doi: 10.22198/rys20I9/3I/II77

* Cátedra CONACYT adscrita al Centro de Investigación en Asistencia en Tecnología y Diseño del Estado de Jalisco (CIATEJ). Av. Normalistas 800, Colinas de La Normal, C. P. 44270, Guadalajara, Jalisco, México. Correo electrónico: Iflores@ciatej.mx

Recibido: 31 de enero de 2019

Aceptado: 18 de octubre de 2019

Liberado: 20 de noviembre de 2019

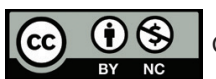
4.0 Internacional. 


\section{Introducción}

La alimentación implica una serie de procesos complejos que permiten al ser humano el acceso a nutrientes para su desarrollo biológico y su reproducción, pero también implica el despliegue de elementos que condensan simbologías y sentidos al hecho alimentario en los niveles social y cultural (Piña, 2014). El proceso para adquirir los alimentos ha sido históricamente un acto trascendental, problemático, complejo y retador. La "domesticación" de los alimentos se considera un hito en la historia de la humanidad: ha permitido contar con productos en cualquier época del año, porque la dependencia a la estacionalidad y a las condiciones climáticas prácticamente se ha reducido y, en algunos casos, nulificado (Contreras, 2005).

Los procesos tecnológicos han dotado a la "domesticación del campo" con infraestructura, evitando pérdidas por eventualidades climáticas y tecnificando los procesos para la producción de alimentos que, bajo otras condiciones, resultarían complicados. Se han incorporado métodos con intervención biológica para potenciar cultivos y mejorar semillas (Becerra y Paredes, 2000; Paliwal, 2001; Vallejo, 2002). No obstante, a pesar de los avances que impactan en la producción de alimentos, nos enfrentamos paradójicamente a la carencia, a la estandarización y a la monopolización de productos. Encontramos algunos ejemplos en las frutas y hortalizas que se han reducido a sólo la producción de determinadas variedades (Durán, 2013), dejando de lado otras, consideradas no comerciales. El mercado ha ocasionado la producción en cadena de algunos productos agrícolas específicos debido a su valor comercial, lo cual ha causado una reducción en la variedad de los cultivos (Morales, Aguilar y Castellanos-López, 2015).

El presente documento tiene como objetivo evidenciar cómo las condiciones globales y sociales en las cuales se enmarca la producción de alimentos en el estado de Chiapas -oscilando entre programas de reconversión productiva para el cultivo de productos comerciales de alta demanda en el mercado- merman los cultivos tradicionales de subsistencia. Se parte de datos generados en el marco de un proyecto realizado junto con el gobierno estatal, ${ }^{1}$ mediante el cual se recolectó información de fuentes secundarias y se realizó trabajo de campo en algunas regiones de Chiapas, incluidas la Frailesca, Metropolitana, Soconusco y la Meseta Comiteca. Se recopiló información a través de entrevistas directas con diversos actores relacionados con la producción agrícola del estado. En este artículo se presentan resultados descriptivos derivados del análisis de los datos del Servicio de Información Agroalimentaria y Pesquera (SIAP) sobre la producción agrícola en Chiapas, que sustenta la discusión relativa a los problemas históricos-sociales que enmarcan el contexto de desigualdad, pobreza, rezago y marginalidad de la población. Muestra los contrastes entre las virtudes geográficas, físicas e hidrológicas y la problemática gestada por la implementación de políticas agrícolas globales que han causado una disminución en la producción de granos básicos, comprometiendo el derecho humano a la alimentación

1 Fondo Mixto núm. 2244, Consejo Nacional de Ciencia y Tecnología (CONACYT) y Gobierno del Estado de Chiapas. 
(Carrera, 1995; Grammont, 2001). Los efectos de las tendencias globales en la producción de alimentos se evidencian en la disminución de superficie sembrada de granos básicos, de frutas y de hortalizas para dar cabida a otros cultivos de mayor valor económico, como la palma africana, el café y ciertos frutales, como mango, papaya y rambután, entre otros. Se observa la contribución de cultivos específicos que aportan un mayor valor en la producción agrícola pero en detrimento de cultivos básicos indispensables para asegurar el derecho de los pueblos a la alimentación.

Para el análisis, se crearon categorías según los cultivos que reporta el SIAP sobre la entidad, considerando alimentos básicos aquellos que sustentan la dieta regional en términos nutricionales y culturales: maíz, frijol, trigo y arroz, y entendiendo como cultivos comerciales (CC) aquellos promovidos por el gobierno como forma de capitalizar a los productores: palma de aceite, café y frutales como mango, plátano y vainilla. Se establecen comparaciones entre 2003, año a partir del cual existen datos abiertos en el SIAP, y 2016, último año en el cual existen reportes completos acerca de la superficie sembrada, de la cosechada y valores de cosecha. Las comparaciones se hacen por región socioeconómica de la entidad, demarcación llevada a cabo por dependencias de gobierno ${ }^{2}$ que delimita la reunión de información por parte de la Secretaría de Agricultura, Ganadería, Desarrollo Rural y Pesca. Además dicha demarcación favorece la identificación de características específicas en términos sociales y culturales de las regiones de Chiapas.

\section{La producción alimentaria como problema global y local. El modelo agrícola predominante y la producción agrícola en Chiapas}

A escala global, la producción alimentaria actual se encuentra sustentada por un modelo de agro-negocio (Acosta, 2006; Cáceres, 2015), caracterizado por su enfoque en la exportación. En este esquema, los precios del mercado internacional dictan las prioridades productivas de acuerdo con criterios de rentabilidad y tendencias generales de consumo en centros urbanos. El cultivo se hace con semillas patentadas, a menudo transgénicas, bajo la noción de monocultivo industrial, intensivo en insumos químicos, mediante paquetes tecnológicos específicos (Poth, 2016; Rosset, 2014).

Este modelo se gestó como parte de la denominada revolución verde en la década de 1960 avalado por organismos internacionales (Barajas, 2015). Para Domínguez (2015), dicha revolución obedeció a una “lógica de reorganización de la agricultura industrial bajo las condiciones de las empresas transnacionales y el capital financiero" (p. 147). Ha solventado la problemática del abasto alimentario (Poth, 2016; Sasson, 2000) y ha tenido una tendencia de crecimiento relevante en algunos países, pero también ha gestado una serie de pro-

2 Gobierno de Chiapas. "Instrumento normativo para la formulación de anteproyecto de presupuesto de egresos". Capítulo XII "Clasificación municipal y regional", 2015. 
blemáticas que incluyen desde el deterioro ambiental como consecuencia del desarrollo de monocultivos por el desgaste de las tierras, la estandarización alimentaria por disminución de variedades, hasta las problemáticas sociales de incremento en la desigualdad por capacidades productivas, lo que se refleja en el abandono del campo, la pobreza y la migración, entre otros problemas (Bartra, 2008; Cuero, 2011; Morales, Aguilar y Castellanos-López, 2015).

En México la producción alimentaria ha tenido una historia compleja de organización por la tenencia y el uso de la tierra, así como por la convivencia de cultivos básicos con cultivos comerciales promovidos por el gobierno en programas de apoyo al campo. Estos programas se basan en tendencias y compromisos comerciales internacionales, como fue el caso de Procampo en 1993, destinado a focalizar recursos subsidiarios a campesinos y pequeños productores rurales de manera individual, para ir abandonando gradualmente el esquema proteccionista y debilitar la propiedad colectiva (Herrera, 2008). No todos los programas de apoyo al campo han tenido continuidad entre sexenios a escala nacional y menos aún en lo estatal, como es el caso de los programas de reconversión productiva para la siembra de agrocombustibles, como el piñón (Jatropha curcas) y la palma africana en Chiapas, en 2008 (Secretaría de Hacienda, 2011; Valero, Cortina y Vela, 2011). Estas estrategias se plantearon como alternativas de obtención de ingresos para productores pobres, ubicados en tierras de bajo potencial productivo (López, 2014), pero experimentaron múltiples vicisitudes para su implementación y, finalmente, dejaron huellas de empobrecimiento entre los productores que las adoptaron y que reconvirtieron sus tierras (Velasco, 1999; Villafuerte, 2015; Solís, 2015).

La política agraria en las décadas de 1960 y 1970 subsidiaba los granos básicos y se enfocaba en el apoyo al campo a través de diversos programas (Bartra, 1972; Quintana, 1995; Warman, 1980 y 2001). El reparto agrario solventó transitoriamente el problema de la tenencia de la tierra, dando legalidad y acceso a tierras de labranza. Aunque estas iniciativas no solucionaron el problema (Delgado, 1965), la producción agrícola empezaba a repuntar (Flores, 1960).

A partir de la década de 1980 las políticas viraron y adoptaron el modelo agroindustrial, abrieron canales de comercialización que se incrementaron con la incorporación al Tratado de Libre Comercio de América del Norte, se disminuyeron los apoyos gubernamentales a cultivos tradicionales para priorizar el apoyo a productos estratégicos con alto valor comercial (Escalante y Talavera, 1998; Lara y Grammont, 1999; Grammont, 2001). Los efectos de las políticas globales relativas a la producción alimentaria han repercutido de forma importante en el incremento de la pobreza, de la desigualdad y del desempleo, despojando de capacidades competitivas a los sectores agrícolas de pequeña escala, debido a las reglas de mercado de los monopolios internacionales (Silva, 2017).

En el caso chiapaneco, las condiciones geográficas develan un papel crucial en la producción alimentaria. Chiapas cuenta con dos ríos principales: Usumacinta y Grijalva, los cuales dividen la entidad en siete áreas biodiversas, distintas en clima, calidad de tierra, actividad económica y demografía (Comisión Nacional para el Conocimiento y Uso de la Biodiversidad [CONABIO], 2013). Existen recursos naturales específicos que generan insumos que no se quedan en la región. 
Chiapas genera aproximadamente $50 \%$ de la energía hidroeléctrica del país, a través de siete presas - La Angostura, Malpaso, Peñitas, Chicoasén, Bombaná, Schoina y José Cecilio Valle (Galicia, 2016)-, pero a su vez se ubica como una de las entidades con menos electrificación; el Instituto Nacional de Geografía y Estadística (INEGI, 2010) la registra como la cuarta entidad con la menor tasa de acceso a la electricidad.

El Programa Estatal de Reordenamiento Territorial de Chiapas (El Colegio de la Frontera Sur, 2005) define la denominada región sursureste. La extensión territorial equivale a $3.8 \%$ del país, posee aproximadamente trescientos kilómetros de litorales y un vasto sistema hidrológico. Estas características permiten "la generación de una mezcla de unidades de paisaje en todas sus superficies y la presencia de 35 tipos de clima" (p. 45). Cuenta con dos grupos climáticos: el cálido-húmedo y el templado-húmedo, cuyas condiciones son propicias para una gran diversidad de ecosistemas. Chiapas se considera el segundo estado de México con la mayor riqueza de flora y fauna, una riqueza biológica dada su localización (p. 10). Además, la diversidad geográfica detona condiciones ambientales favorables para la producción de materias primas.

No obstante, Chiapas es uno de los estados con mayor índice de pobreza. Datos del INEGI reportan que $74 \%$ de la población se encuentra en la pobreza y $46 \%$ en la categoría de extrema pobreza asociada a la condición indígena (INEGI, 2010). El "Censo de población y vivienda 2010" refiere que $27 \%$ de la población de la entidad habla una lengua indígena, 12 de los 62 pueblos reconocidos oficialmente indígenas se ubican en Chiapas y más de $51 \%$ de la población vive en zonas rurales donde se desarrolla de manera predominante la producción primaria de alimentos (Villafuerte-Solís, 2015).

La agricultura representa el principal motor de la economía chiapaneca. Según el Sistema de Información Agrícola y Pecuaria (SIAP-SAGARPA, 2016-2018), se cultivan 53 productos cíclicos y perennes. La extensión territorial del estado se acerca a las 7.5 millones de hectáreas, pero para el cultivo sólo se utilizan 1.4 millones de hectáreas, 20\% del total (Comisión Nacional Forestal, 2010). Se identifican dos formas de producción: la agricultura comercial y la de subsistencia. La primera remite a procesos de tecnificación, se desenvuelve en las regiones Soconusco y Centro, con cultivos vinculados a la exportación, como café y plátano. En el mercado nacional, el maíz ha tenido un papel relevante, aunque con modificaciones importantes y con poca infraestructura agrícola. La segunda incorpora procesos intensivos de mano de obra, como el familiar, y su producción es en primer lugar para el autoabasto; se reservan para la venta sólo los excedentes (Fundación Produce, 2011; Solís, 2015).

A partir de 2007, se han promovido programas de reconversión productiva en el estado, tanto de cultivos energéticos -el hule, el piñón y la palma de aceitecomo de frutales - plátano, mango, papaya, rambután, mangostán, carambolo y cítricos-. Los datos develan cómo la producción estratégica de cultivos comerciales gana terreno sobre la agricultura de subsistencia (Morales, Aguilar y Castellanos-López, 2015; Valero, Cortina y Vela, 2011; Vidal, Ocón y Cabañas, 2019). Y es que se ha favorecido la producción de monocultivos, que sin duda tienen gran valor comercial, pero que generan una serie de consecuencias ad- 
versas en el sector agropecuario, tanto en lo ambiental como en lo social, entre los que destacan la pérdida de soberanía alimentaria al disminuir la diversidad de productos obtenidos del entorno agrícola, la descapitalización de los productores en caso de que el cultivo comercial no resulte redituable, el abandono del campo, el desplazamiento de la diversidad, el deterioro ambiental, la migración, el desempleo, el incremento de la pobreza y mayor inseguridad alimentaria (Cuero, 2011; Morales, Aguilar y Castellanos-López, 2015; Velasco, 1999; Valero, Cortina y Vela, 2011).

En Chiapas las superficies sembradas en 1996 eran 1410937 hectáreas, en las que se reportan 39 cultivos. Los principales eran café, caña de azúcar, maíz, mango, cacao, soya y frijol. Las superficies sembradas en 2016 fueron de 1422215 hectáreas y tuvieron 63 cultivos genéricos según el SIAP, que representaban $19 \%$ de la extensión territorial. Datos de esta misma instancia refieren una reducción en 2016 de 1.4\% de la superficie sembrada respecto a 1443526 hectáreas sembradas en 2013. Si bien hubo una reducción en las superficies sembradas, el valor de la producción aumentó. Se reporta un crecimiento de 9.28\% (SIAP, 2016). El valor de la producción de café y palma de aceite ocupó los primeros lugares.

Se considera que la producción de café es la actividad agrícola más consolidada en el país, con una historia de más de 157 años. Ha generado una demanda económica en divisas y fuerza de trabajo para sus fases de producción. Chiapas continúa siendo el primer estado productor de café a escala nacional. Ochenta por ciento de su producción se destina a los mercados internacionales y contribuye, junto con Oaxaca, Veracruz, Puebla, Guerrero, Hidalgo, Nayarit y San Luis Potosí, a ubicar México en el decimoprimer lugar de producción de café a escala mundial. Además, es el primer productor de café orgánico (SIAP, 2017). El cultivo de café ha atravesado por diversas crisis, particularmente relacionadas con plagas y enfermedades, así como afectación de plantaciones por cambios climáticos. Sin embargo, continúa siendo un producto estratégico en las regiones chiapanecas Soconusco, Sierra Mariscal y Tulija Tseltal Chol.

La palma de aceite ha pasado a ser relevante debido a la generación de valor agregado en los subproductos encaminados a la producción de biocombustibles y tiene implicaciones importantes en términos sociales y ambientales (Fletes, Rangel, Velas y Ocampo, 2013; Herrera, 2009; Landívar, López y Yela, 2013). México ocupa el lugar 77 en la producción a escala mundial. En América Latina se ubica en el cuarto lugar. El cultivo de la palma se introdujo en el campo chiapaneco en la década de 1990 y ha sido promovido por los programas de reconversión productiva impulsados por el gobierno estatal en 2000. En 2017, Chiapas fue uno de los principales productores a escala nacional junto con Campeche, Tabasco y Veracruz (SIAP, 2017).

La relevancia en la producción del mango radica en un incremento en las exportaciones como un reflejo del aumento en el consumo. Álvarez (2001) señala que el consumo de mango en Estados Unidos se elevó entre 1989 y 1995 en $216 \%$. En Chiapas las dos regiones productoras son Istmo-Costa y Soconusco. Según datos de García y Morales (2017), es en 1965 cuando se establece la primera plantación comercial de mango. Los datos de SIAP-SAGARPA (2017) 
reportan que Chiapas tuvo un volumen de producción de 264827 toneladas, lo cual ubica al estado como el tercer productor de mango a escala nacional. Las principales variedades que se producen son: Ataúlfo, criollos, manililla y Tommy Atkins. Las dificultades que enfrenta la producción de mango en la región son las plagas, las enfermedades, el uso excesivo de agroquímicos que afectan incluso la producción, la falta de infraestructura, la venta a bajos precios por parte de pequeños productores a acopiadores, el acaparamiento por parte de los empresarios para comercializar, la inexistencia de valor agregado de los productos, la incipiente industria de deshidratación y la venta de fruta a bajos precios, por parte de algunos productores, a la industria alimentaria para la elaboración de jugos (Entrevistas, trabajo de campo, 2018).

El consumo de papaya se ha incrementado a escala mundial por sus propiedades terapéuticas (Juárez, 2013). México es un productor importante de papaya, particularmente de la especie maradol. Oaxaca, Colima y Chiapas son las principales entidades productoras. A escala nacional, en el período 2012-2018, se evidenció un aumento anual promedio de $6.2 \%$, considerado el mayor volumen cosechado históricamente, con 961000768 toneladas. Sin embargo, la variación en porcentaje en este período, para el caso chiapaneco, fue negativa según el Atlas agroalimentario (SAGARPA, 2018). La producción en Chiapas se desarrolla en las regiones Metropolitana, Valles Zoques, De Los Llanos, Frailesca, Soconusco y Sierra Mariscal, y aportó en conjunto un volumen promedio de producción de 10393.64 toneladas en 2017 (datos abiertos del SIAP, 2017). La papaya mexicana sigue posicionada en el quinto lugar a escala mundial. Su principal destino son los mercados internacionales, concretamente Estados Unidos.

Tabla 1. Indicadores agrícolas de cultivos comerciales en Chiapas

\begin{tabular}{|l|r|r|r|r|r|r|}
\hline & \multicolumn{3}{|c|}{2003} & \multicolumn{3}{|c|}{2017} \\
\hline Cultivo & $\begin{array}{c}\text { Superficie } \\
\text { sembrada } \\
\text { (ha) }\end{array}$ & $\begin{array}{c}\text { Volumen de } \\
\text { la producción } \\
\text { (ton) }\end{array}$ & $\begin{array}{c}\text { Valor de la } \\
\text { producción } \\
\text { (pesos) }\end{array}$ & $\begin{array}{c}\text { Superficie } \\
\text { sembrada } \\
\text { (ha) }\end{array}$ & $\begin{array}{c}\text { Volumen de } \\
\text { la producción } \\
\text { (ton) }\end{array}$ & $\begin{array}{c}\text { Valor de la } \\
\text { producción } \\
\text { (pesos) }\end{array}$ \\
\hline Café cereza & 241144 & 587765 & 1548473160 & 254845 & 339361 & 1788167409 \\
\hline Mango & 22493 & 130686 & 398233713 & 38741 & 264827 & 1297822049 \\
\hline $\begin{array}{l}\text { Palma africana } \\
\text { o de aceite }\end{array}$ & 16793 & 200492 & 150036924 & 44465 & 500783 & 778469559 \\
\hline Papaya & 2575 & 165593 & 619255530 & 1991 & 145511 & 691568412 \\
\hline Plátano & 23757 & 709406 & 771169839 & 23455 & 688900 & 1667387492 \\
\hline Total general & 306762 & 1793942 & 3487169166 & 363496 & 1939382 & 6223414921 \\
\hline
\end{tabular}

Fuente: elaboración propia. Datos abiertos del SIAP, 2003-2017.

Es evidente el valor comercial de estos cultivos y el impulso que se les ha dado. Se observa que las políticas económicas y del desarrollo del campo en México han focalizado, impulsado y promovido su avance a través de programas específicos, como el de reconversión productiva de palma de aceite, el de Impulso Productivo al Café, el de PROCAFÉ, el de Impulso Emprendedor 
y el de Desarrollo de Zonas Áridas (PRODEZA), entre otros. Estos cultivos se constituyen en objeto relevante por el valor económico que representan. Sin duda el panorama que se muestra está ligado a las tendencias de consumo y obedece a un modelo agroindustrial que poco empata con las condiciones sociales y culturales de los pueblos (Valero, Cortina y Vela, 2011; Morales, Aguilar y Castellanos-López, 2015; Vidal, Ocón y Cabañas, 2019).

\section{La tensión entre la reconversión productiva de cultivos comerciales y la soberanía alimentaria}

La alimentación es un derecho humano que el Estado está obligado a garantizar, de acuerdo con los artículos cuarto y vigesimoséptimo de la Constitución Política de los Estados Unidos Mexicanos (2019), en términos de acceso físico y económico, para lo cual la producción agrícola nacional y la local se convierten en elementos determinantes. La autonomía de los pueblos para decidir las condiciones bajo las cuales determinan su producción agrícola -lo que se cultiva y cómo se cultiva, partiendo de sus propios criterios culturales y sociales- se ha definido como soberanía alimentaria (Martínez, 2010; Rosset, 2008; Rosset y Torres, 2016). Se establece como lucha del movimiento campesino ante el embate de las grandes corporaciones que monopolizan la producción alimentaria en el orbe (Rosset, 2014).

La noción de soberanía está en contraposición con la política asumida por el Estado y respaldada por organismos internacionales que instituyen la seguridad alimentaria como vía para garantizar el acceso a los alimentos y erradicar el hambre en el mundo (FAO, 2003). La dificultad de esta visión radica en no involucrarse en la polémica sobre las formas de producción de alimentos, pues se focaliza en proporcionarlos sin considerar las condiciones que sustentan su producción. La política social de combate a la pobreza en la región ha sido cuestionada por su estrategia de proporcionar alimentos densamente calóricos, con poco contenido nutricional, que implican altos costos de traslado y que favorecen a ciertos sectores de la industria alimentaria (Becerra y Ortega, 2015; Rodarte, 2015; Salazar, 2014), y se suma a ello la falta de apoyo en la producción de alimentos de calidad.

La agricultura campesina ha desempeñado un papel relevante, porque ha permitido a los pueblos producir para el autoconsumo (Colín, Cuevas y Tradicional, 2015; Chablé-Pascual et al., 2015; Nicholls y Altieri, 2011), una forma de soberanía alimentaria implementada histórica y culturalmente a escala local-regional, ${ }^{3}$ consistente en la siembra de cultivos básicos a partir de variedades loca-

3 La noción "local-regional" remite al territorio como un espacio que se manifiesta en la formación de subsistemas donde convergen características comunes en términos geográficos, sociales, culturales e históricos (Lira, 2003). Saldaña y Sánchez (2010) definen lo local como una espacialidad de la existencia de lo social en una menor escala de lo regional. Se puede pensar la diferencia entre lo regional no sólo en términos de mayor amplitud espacial sino también en términos de las dinámicas sociales y culturales que emergen y difieren en los espacios locales (Bautista y Yáñez, 2001). En este documento se presenta un análisis regional que obedece a una clasificación gubernamental e incluye diversos municipios y localidades. Sin embargo, hacemos uso del término "local" porque, a pesar de que éstos se encuentran en el mismo espacio regional, difieren de este último en sus dinámicas sociales y culturales. 
les, para el autoconsumo, que ha sido desmantelada como parte de las políticas macroeconómicas (Escalante y Talavera, 1998; Viñas, 2013).

Fletes, Guzmán y Ocampo (2016) reportan injerencia de la administración estatal del período 2006-2012 en el impulso a la palma africana, el piñón, el limón y el aguacate, entre otros productos. Los cultivos que se han promovido en los últimos sexenios distan mucho de garantizar el abasto de granos básicos para satisfacer una alimentación culturalmente adecuada (de acuerdo con los elementos socialmente aceptables para la dieta). Además de la reconversión productiva hacia agrocombustibles en las regiones 10 (Soconusco) y 13 (Maya), se ha enfatizado en el uso de los frutales para zonas cálido-húmedas, como el Soconusco y la Selva, en el entendido de lograr la rentabilidad de la tierra. Sin embargo, actualmente las cadenas productivas se encuentran en manos de intermediarios, quienes sacan el mayor provecho de la producción agrícola. Los cultivos de granos básicos han disminuido a partir de los programas de reconversión productiva hacia cultivos comerciales, lo cual ha repercutido en la devastación de las agriculturas familiares y ha dado paso a la desestructuración de las unidades campesinas (Gómez, 2017).

La reconversión productiva en el estado se promovió bajo la premisa de un mayor ingreso para la compra de alimentos, dejando de lado la autosuficiencia en la producción de alimentos básicos; es decir, la lógica fue sembrar cultivos que redituaran a los productores con ingresos suficientes para comprar la canasta básica y productos externos a la unidad productiva. Pese a que hubo cultivos, como el de la Jatropha o piñón, que inicialmente se promovieron como de convivencia con el maíz y el frijol, ese postulado se probó incorrecto a los cuatro años de siembra (López, 2014), al comprobarse la alta competencia por los recursos entre cultivos, lo que incrementó costos por la aplicación de fertilizantes. La reconversión ha implicado la sustitución de "áreas dedicadas a la producción de alimentos básicos para el fomento de cultivos comerciales" (Escalante, Catalán, Galindo y Reyes, 2014, p. 19). En Chiapas subsistió la palma africana como cultivo prioritario para la elaboración de agrocombustibles (Villafuerte y García, 2014).

La reconversión productiva como un elemento que detonaría el desarrollo de los pueblos, no ha sido evidenciada. Guzmán et al. (2008) describen los efectos negativos en la producción. Ellos refieren las siguientes situaciones que enfrenta el sector primario: "problemas financieros, ausencia de tecnología e infraestructura, falta de capacitación y organización adecuada para la comercialización, altas mermas, problemas propios del cultivo derivado del entorno y los cambios ambientales" (p. 133). Valencia, Duana y Hernández (2017) concluyen en su análisis de competitividad para uno de los cultivos estratégicos que los problemas del sector primario no se han resuelto y esto trae como consecuencia una disminución en el producto interno bruto (PIB) agroalimentario.

\section{Aproximación metodológica}

Se muestra información respecto a la dinámica productiva en un lapso de trece años para evidenciar los impactos de la producción en cultivos estratégicos y 
la disminución de productos básicos. Se analizan los datos abiertos derivados del SIAP -correspondientes a 2003 y a 2016-, sobre 15 regiones definidas como unidades económicas según el gobierno de la entidad (véase figura 1). Se clasificaron cultivos básicos y cultivos comerciales. Los primeros incluyen aquellos que son parte importante tanto nutricional como culturalmente en la dieta de los habitantes de la región. Se incluyeron en esta clasificación el maíz, el frijol, el trigo y el arroz. Se consideraron cultivos comerciales aquellos que tienen un valor comercial en mercados externos. Entre ellos se encuentran la palma de aceite y el café. En el rubro de frutales, que se consideran en la clasificación de cultivos comerciales, están incluidos el mango, el plátano y la vainilla. Los criterios para la clasificación son limitados y partimos de los datos abiertos del SIAP.

Figura 1. Regiones económicas en Chiapas

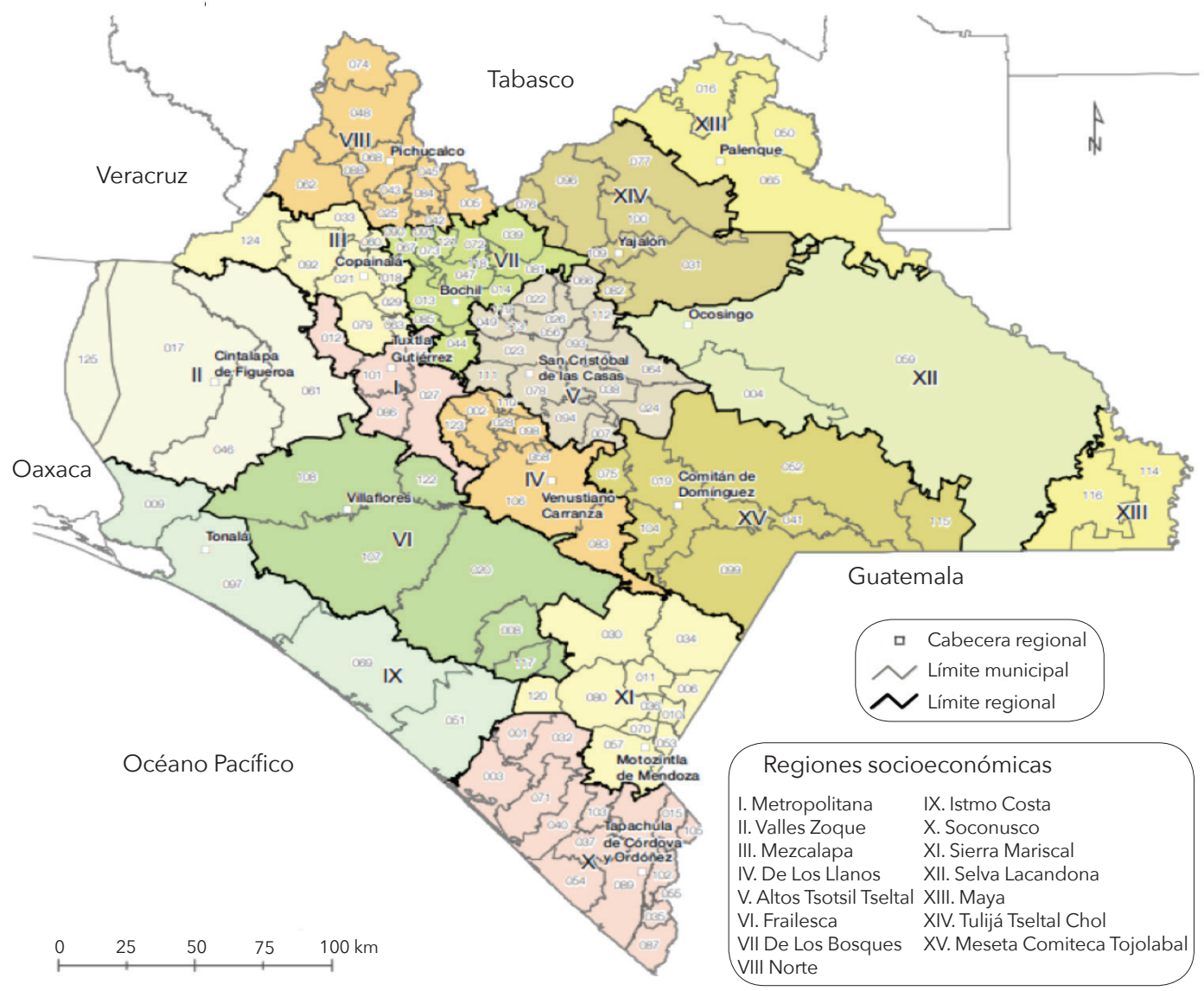

Fuente: Constitución Política del Estado de Chiapas (2017).

\section{Cambios en la producción de cultivos agrícolas}

Describimos las características productivas de los municipios que se ubican en condiciones de mayor pobreza según el Consejo Nacional de Evaluación de la 
Política de Desarrollo Social (CONEVAL). Éstos son Aldama, San Juan Cancuc, Chalchihuitán y Santiago del Pinar, ubicados en la región 5, así como el municipio de San Andrés Durazal, que se encuentra en la región 7. En los casos de estos municipios, en 2003 y en 2016 se observa la producción sostenida de café y de maíz. No obstante, en Santiago del Pinar se observa una reducción en la superficie sembrada y cosechada de maíz en 2016 de casi la mitad de las hectáreas cultivadas: se pasó de 554 a 290 hectáreas registradas en el lapso de un año (véase figura 2).

Figura 2. Promedio de superficie sembrada por región económica de Chiapas

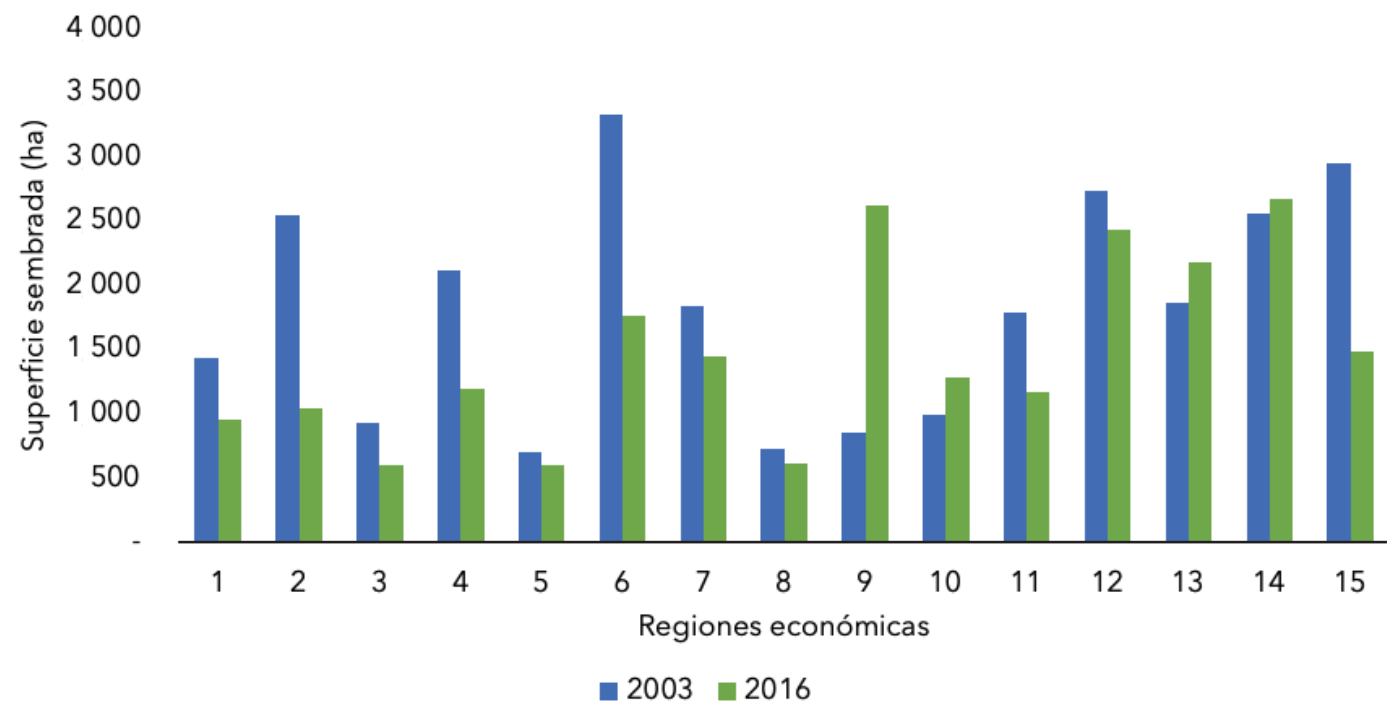

Fuente: elaboración propia a partir de datos abiertos de SIAP-SAGARPA, 2003 y 2016.

Se clasificaron cultivos comerciales, incluyendo café, palma de aceite, mango, plátano y vainilla, y se comparan con la producción de frutas y hortalizas, excluyendo las que se ubicaron como parte de los cultivos comerciales. Se integró una categoría para granos que incluyó frijol, maíz, trigo y arroz. Se presentan las comparaciones entre la producción reportada en 2003 y 2016, evidenciando una caída drástica en la superficie sembrada de granos básicos en las regiones 1, 2, 4, 5, 6 y 13, correspondientes a las regiones Metropolitana, Valles Xoque, De Los llanos, Altos Tsotsil-Tseltal, Frailesca y Maya, respectivamente. Hubo un repunte en la región 13 en 2016. En términos generales y de manera comparativa, la disminución de la superficie sembrada en este período es considerable (véase figura 3).

La dinámica predominante entre todas las regiones, tanto en términos de cultivos generales como de granos básicos, es una disminución de la superficie 
sembrada con granos básicos en 2016 con respecto a 2003. La figura 3 ilustra de forma comparativa la dinámica en 2003 y 2016 con respecto a la superficie sembrada (ha) de la producción agrícola por regiones económicas. La disminución de los cultivos agrícolas en 2016 es relevante.

Figura 3. Promedio de superficie sembrada de granos básicos por región económica, Chiapas

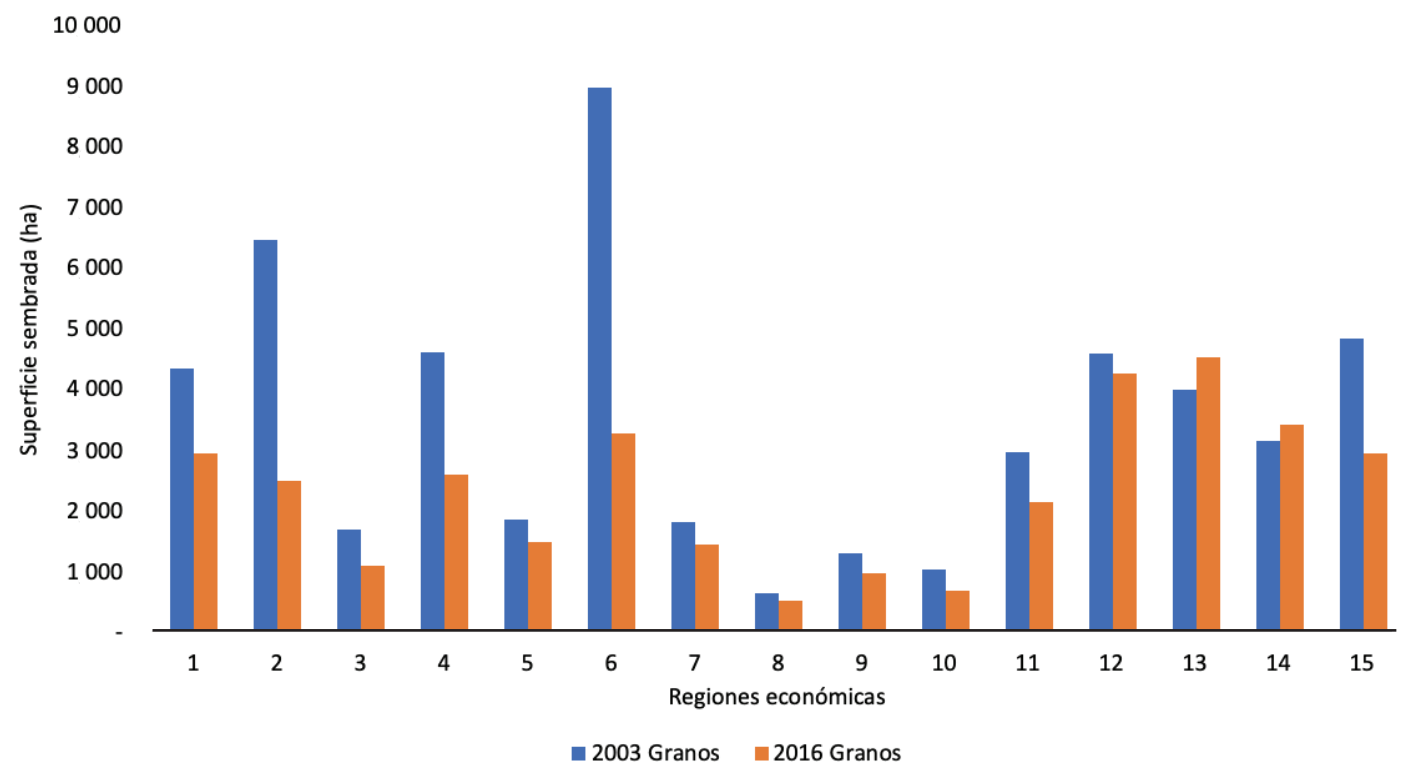

Fuente: elaboración propia a partir de datos abiertos de SIAP-SAGARPA, 2003 y 2016.

Analizando los datos relativos a la superficie sembrada, cosechada y al valor de la producción, se observa una disminución en la superficie sembrada y cosechada en la mayoría de las regiones, aunque el valor de la producción se incrementa en la región 1, 4, 6, 10 y 15, región Metropolitana, De Los llanos, Frailesca, Soconusco y Meseta Comiteca, respectivamente. Sin embargo, disminuyó la producción de granos básicos.

Tabla 2. Comparación de promedios de superficie sembrada, cosechas y valor de la producción por región económica en Chiapas

\begin{tabular}{|l|c|c|c|c|}
\hline & \multicolumn{2}{|c|}{2003} & \multicolumn{2}{c|}{2016} \\
\hline Región & $\begin{array}{c}\text { Superficie } \\
\text { Sembrada } \\
\text { (ha) }\end{array}$ & $\begin{array}{c}\text { Valor de la } \\
\text { producción } \\
\text { (pesos) }\end{array}$ & $\begin{array}{c}\text { Superficie } \\
\text { sembrada } \\
\text { (ha) }\end{array}$ & $\begin{array}{c}\text { Valor de la } \\
\text { producción } \\
\text { (pesos) }\end{array}$ \\
\hline 1. Metropolitana & 1325 & 4881419 & 962 & 9400655 \\
\hline 2. Valles Zoque & 2401 & 11043288 & 1042 & 9797532 \\
\hline 3. Mezcalapa & 928 & 1827309 & 599 & 3733319 \\
\hline
\end{tabular}




\begin{tabular}{|c|c|c|c|c|}
\hline 4. De los Llanos & 1965 & 15851381 & 1202 & 22283317 \\
\hline 5. Altos Tsotsil-Tseltal & 711 & 2537537 & 599 & 6117715 \\
\hline 6. Frailesca & 3053 & 13059311 & 1580 & 21022769 \\
\hline 7. De los Bosques & 1847 & 5476293 & 1449 & 9435984 \\
\hline 8. Norte & 729 & 2832068 & 615 & 8510644 \\
\hline 9. Istmo-Costa & 2501 & 57390499 & 2252 & 32798034 \\
\hline 10. Soconusco & 1157 & 17157383 & 1219 & 25873055 \\
\hline 11. Sierra Mariscal & 1795 & 9750729 & 1173 & 12210638 \\
\hline 12. Selva Lacandona & 2736 & 7344962 & 2431 & 12307391 \\
\hline 13. Maya & 1868 & 5495371 & 2027 & 14976910 \\
\hline 14. Tuljá Tseltal Chol & 2562 & 7203903 & 2673 & 14855311 \\
\hline 15. Meseta Comiteca Tojolabal & 2957 & 17330803 & 1483 & 23467883 \\
\hline Total general & 1642.6 & 11653622 & 1285 & 16017554 \\
\hline
\end{tabular}

Fuente: elaboración propia a partir de datos abiertos de SIAP-SAGARPA, 2003 y 2016.

En relación con los cultivos comerciales, en este período se observa en general la tendencia al incremento de las superficies sembradas, particularmente en las regiones 5, 6, 9 y 13, correspondientes a Altos Tsotsil-Tseltal, Frailesca, Istmo-Costa y Maya, respectivamente. Un dato relevante es que la producción en la Meseta Comiteca (región 15) presentó una disminución considerable, tanto en las superficies sembradas de granos básicos como en las de cultivos comerciales (véase figura 4).

Figura 4. Promedio de superficie sembrada de cultivos comerciales por región económica, Chiapas

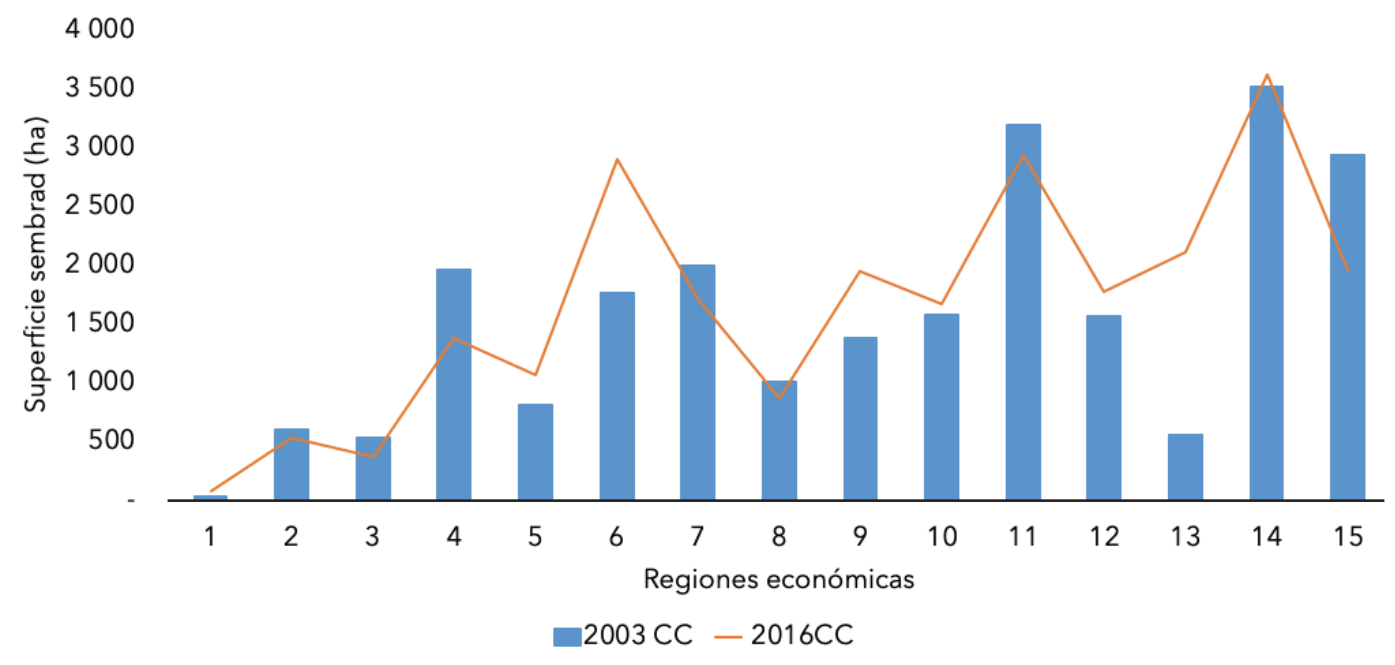

Fuente: elaboración propia a partir de datos abiertos de SIAP-SAGARPA, 2003 y 2016. 


\section{Discusión}

Se ha visto cómo se ha modificado la producción de los principales recursos agrícolas en el estado de Chiapas partiendo de la información abierta y disponible del SIAP. Se ha podido observar una mayor producción de cultivos comerciales en contraposición con los cultivos básicos. En el caso de la región 5 , donde se ubica uno de los municipios con mayor pobreza y rezago social, se evidencia la disminución en la producción de granos básicos, según la clasificación que se propuso. La discusión respecto a la promoción y al apoyo de determinados cultivos no es en términos de mermar o nulificar la producción de productos agrícolas comerciales, sino que se cuestiona la reducción de las áreas de cultivo dedicadas a la producción de granos básicos que permiten la subsistencia de una gran proporción de la población que hace uso de estos insumos. Villafuerte (2010) señala un descuido en la producción de autoconsumo y la mayor dependencia del mercado. La producción de cultivos para autoconsumo resolvía en gran medida la disponibilidad de alimentos que podían ser integrados a la dieta diaria y que permitían la reproducción social. Eliminar esta vía potencializa la crisis alimentaria en la región. Colín, Cuevas y Tradicional (2015) encuentran que en los cultivos de traspatio son favorecidas aquellas especies que tienen valor comercial. Sin embargo, en su estudio también encuentran que se conservan vegetales con importancia cultural, destinados al autoabasto. Chablé-Pascual et al. (2015) encontraron un total de 330 especies de vegetales y 17 especies de fauna como parte de un estudio de biodiversidad en huertos familiares en la región sureste. Monroy, Colín, Cruells, Flores y Ayala (2016) y Monroy, Ponce, Colín, Monroy O. y García (2017) caracterizan la riqueza de especies como parte de los huertos de traspatio y como ejemplo de agro-ecosistemas con producción sostenida. García, Gutiérrez, Balderas y Araujo (2016), Flores (2017) y Sánchez y Martínez (2016) evidencian cómo la práctica tradicional campesina del huerto familiar se constituye en una estrategia que contribuye de forma sustantiva a la soberanía alimentaria. Identifican 134 especies, de las cuales 54 son hortalizas. Se utilizan como parte de la alimentación y como condimentos. Además, los campesinos les otorgan un uso medicinal y las emplean en rituales. Se destinan concretamente para autoconsumo y los excedentes, a la venta e intercambio, como otras formas de autosuficiencia alimentaria.

La soberanía alimentaria ha sido un recurso que ha permitido la sobrevivencia de la gente de estas regiones (Boza Martínez, 2010; Martínez Castillo, 2010). Se ha implementado de antaño a través de la diversificación de cultivos, la producción de autoconsumo, la red local de intercambio y el trueque, entre otros mecanismos que han sido imperativos como parte de la dinámica social y cultural.

Román-Ruiz y Hernández (2010) analizan los medios de vida de las familias en Chiapas. Los hallazgos de su estudio son relevantes porque evidencian que en esas microrregiones, ${ }^{4}$ Tzay, Pasthtonticjá y Oxchuc-centro, pertenecientes al municipio de Oxchuc, correspondientes a la región 5, la producción tradicional

4 Los autores incorporan el término "microrregión", porque el municipio de Oxchuc estaba dividido en unidades territoriales de desarrollo según la clasificación del Sistema de Desarrollo Social (SEDESOL). En su análisis se acogen a esta clasificación. 
del sistema milpa (roza-tumba-quema) tiene un escaso rendimiento, pero que sin embargo logra abastecer a las familias. El papel que desempeña la producción de café, si bien genera mayores ingresos, las fluctuaciones en sus precios impactan negativamente al ingreso familiar. Los programas de asistencia social para disminuir la pobreza tienen una función importante en el ingreso familiar. Los autores muestran cómo la economía doméstica de esta región depende de factores externos. Lo más sustantivo del estudio refiere que el gasto en alimentos es mayor en aquellas zonas donde la producción de café abarca una proporción relevante en tierras de cultivo. En las regiones donde la diversificación de cultivos es mayor, la compra de alimentos es menor. Realiza un análisis de diversidad dietética que permite apreciar el tipo de alimentos de compra y concluye que la cantidad calórica proviene de alimentos densamente calóricos en aquellas zonas donde la producción agrícola comercial es la principal actividad económica.

Uno de los problemas más relevantes gira en torno al acceso alimentario en términos de calidad. El ingreso económico de estas comunidades, derivado de la producción agrícola comercial, no garantiza el acceso a alimentos de calidad nutricional. Román-Ruiz y Hernández-Daumas (2010) refieren que las regiones que reciben apoyos gubernamentales tienen una ingesta de mayor cantidad de productos con alta densidad calórica, como harinas y productos chatarra. El apoyo gubernamental depende también de la ubicación del municipio. En las regiones cuyos habitantes consumen alimentos que ellos mismos producen, sólo se compran alimentos para complementar la dieta. La diversidad dietética, en términos de calidad de los alimentos, particularmente de vitaminas y minerales, está cubierta en mayor medida en las regiones que diversifican su producción agrícola.

La noción de seguridad alimentaria enmascara una problemática compleja de acceso alimentario. Esta discusión ha sido abordada por diversos autores (Domínguez, 2015; Rodarte, 2015; Rosset, 2014) en términos de autosuficiencia o soberanía ${ }^{5}$ alimentaria; si bien el ingreso monetario sigue siendo precario, por lo menos estas regiones tenían acceso a la producción de autoconsumo.

La reconversión productiva ha desempeñado un papel crucial en la producción de alimentos. Obedece a una lógica propia del modelo neoliberal, que impulsa mayor ingreso para la compra, no para la autosuficiencia. La estrategia en la producción de cultivos estratégicos, focalizada en el mercado externo, ha provocado consecuencias adversas a la de por sí doliente situación del campo en Chiapas. Valero, Cortina y Vela (2011) describen, en el caso del cultivo de piñón para la producción de biodiesel, cómo se crearon instancias y se movilizaron recursos económicos importantes para la introducción de este cultivo en la región, abandonado en la actualidad (López, 2014; Valero, Cortina y Vela, 2011).

5 Flores, Vázquez y Quintero (2012), López y Franco (2015), Gómez-Trujillo, Martínez-Andrade, Rivas-García y Villalobos-Maradiaga (2016) hacen referencia a la autosuficiencia alimentaria y otros a la soberanía. Podrían considerarse sinónimos, aunque la soberanía puede entenderse como aquello que sustenta la autoridad, quien tiene el poder o en quien reside éste. En este caso, se asume que el pueblo tendría este papel. La autosuficiencia es definida como el estado o condición de quien se basta a sí mismo, independientemente del sustento del poder (Domínguez, 2015; Méndez y Gordillo, 2013). 
Por otro lado, Villafuerte (2010) sostiene que hay un cambio en la geografía del maíz asociado al régimen de subsidios que desalienta la producción de áreas altamente productivas. La parte destinada al fomento productivo es menor; se destinan los subsidios a programas asistencialistas (Romero, 2016). En Chiapas, el recurso a los programas de asistencia concentra más de la mitad del presupuesto asignado en el año 2014. Carrera (1995) señala que a varias décadas de la implementación del modelo económico no existe capitalización agraria ni bienestar social; tampoco se vislumbran ventajas competitivas ni soberanía alimentaria si no se procura el fortalecimiento de políticas agrícolas internas que fortalezcan la economía local.

\section{Conclusiones}

Las condiciones naturales del territorio del estado proveen características apropiadas para la producción de una amplia variedad de cultivos; sin embargo, sólo se han impulsado los cultivos con alto valor económico a través de la producción agroindustrial para la exportación. Se mostró cómo se ha incrementado la producción de productos como café, plátano, cacao y palma de aceite, en contraposición con los de granos básicos. Evidenciamos que existe un impulso a la producción agrícola en Chiapas focalizado en los cultivos estratégicos de importancia para el mercado externo, incluidos en el impulso los recursos y los apoyos gubernamentales. En este tenor, es incuestionable la disminución de cultivos con relevancia social en la región. La diversificación de productos ha disminuido según la información que se deriva de instancias oficiales. Existe una serie de cultivos que no se impulsa y que no se desarrolla por falta de demanda externa (nacional e internacional), aunque desde siempre haya tenido un papel fundamental en la alimentación de pequeñas unidades familiares.

Chiapas se caracteriza por concentrar una población étnica en condiciones de pobreza. Aunque ésta tiene una actividad agrícola relevante, la política gubernamental de apoyar la producción de cultivos comerciales como un mecanismo para mejorar la economía de estas regiones, no ha tenido resultados satisfactorios. La política social ha destinado estrategias sin mucho éxito para el combate a la pobreza a través de diversos programas de índole asistencialista, incluidos los programas de atención al campo. Como resultado se ha generado un incremento de la desigualdad social y de la pobreza, y se ha limitado la soberanía alimentaria que estos pueblos ejercían desde antaño.

La reconversión productiva en el plano económico y la competitividad empresarial se ve como la vía para disminuir la inequidad social. Se dejan atrás varios sistemas productivos regionales para dar paso a otros cultivos comerciales. Sin embargo, las condiciones de rezago en las que se encuentra el estado no han mejorado. Estas iniciativas han limitado los sistemas productivos regionales, han deteriorado las condiciones naturales y han reducido los vastos recursos con los que la entidad cuenta. La producción agrícola promovida en Chiapas se ha focalizado en cultivos estratégicos en términos de mercado externo. Los recursos y el apoyo gubernamental también se han concentrado en el impulso de estos 
cultivos. Es evidente la disminución de cultivos de importancia social en la región. La diversificación de productos ha disminuido, según el registro de fuentes oficiales. Existe una serie de cultivos que no se impulsa ni se desarrolla y que sin embargo han contribuido sustantivamente a la autosuficiencia alimentaria, así como a la reproducción social de los pueblos. Las políticas públicas respecto de la producción de alimentos deben atender las condiciones locales para generar impulso económico en la región. La soberanía alimentaria debe impulsarse como vía de salida a la pobreza, a la desigualdad y a la merma de alimentos, con miras al desarrollo social de estas regiones en Chiapas.

\section{Referencias}

Acosta Reveles, I. L. (2006). Balance del modelo agroexportador en América Latina al comenzar el siglo XXI. Mundo Agrario, 7(3), 25. Recuperado de https: / / www.redalyc.org/articulo.oa?id=84501301

Álvarez, R. R. (2001). Más allá de la frontera: penetración del Estado-Nación, TLC, y control externo en la industria México-americana del mango. Estudios Fronterizos, 2(4), 115-137. Recuperado de http:/ / www.scielo.org. mx/scielo. php?script=sci_arttext\&pid=S0187-69612001000200004\&lng=es\&tlng=es

Barajas, R. E. (2015). Biotecnología y revolución verde: especificidades y divergencias. Sociológica, 6(16), 5-18. Recuperado de http://www.scielo.org. $\mathrm{mx} /$ scielo.php?script=sci_arttext\&pid=S0187-69612001000200004\&lng=es\&t lng=es

Bartra, A. (2008). Fin de fiesta: el fantasma del hambre recorre el mundo. Argumentos, 21(57), 15-31.

Bartra, A. (1972). Campesinado y poder político en México: un modelo teórico. Revista Mexicana de Sociología, 34(3/4), 659-684. doi: 10.2307/3539254

Bautista, J. J. R., y Yáñez, R. C. (2001). Lo global y local, una relación para hacer regiones en el ámbito mundial. Economía y Sociedad, 6(9), 47-66.

Becerra, M. M. Z., y Ortega, A. R. C. (2015). Pobreza alimentaria y política pública en México: un análisis de la Cruzada Nacional Contra el Hambre en León. Jóvenes en la Ciencia, 1(2), 828-832.

Becerra, V., y Paredes, C. (2000). Uso de marcadores bioquímicos y moleculares en estudios de diversidad genética. Agricultura Técnica, 60(3), 270-281.

Boza Martínez, S. (2010). Desafío del desarrollo: la agricultura orgánica como parte de una estrategia de mitigación de la pobreza rural en México. Nóesis. Revista de Ciencias Sociales y Humanidades, 19(37), 92-111. Recuperado de https: / / www. redalyc.org/articulo.oa?id=85919842004

Cáceres, D. M. (2015). Tecnología agropecuaria y agronegocios: la lógica subyacente del modelo tecnológico dominante. Mundo Agrario, 16(31), 1-15.

Chablé-Pascual, R., Palma-López, D. J., Vázquez-Navarrete, C. J., Ruiz-Rosado, O., Mariaca-Méndez, R., y Ascensio-Rivera, J. M. (2015). Estructura, diversidad y uso de las especies en huertos familiares de la Chontalpa, Tabasco, México. Ecosistemas y recursos agropecuarios, 2(4), 23-39. 
Carrera, V. S. (1995). Ni autosuficiencia alimentaria ni ventajas comparativas: los saldos del neoliberalismo en granos básicos y oleaginosas. Cuadernos Agrarios, 5(11-12), 9-66.

Colín, H., Cuevas, A. H., y Tradicional, R. E. M. (2015). El manejo tradicional y agroecológico en un huerto familiar de México, como ejemplo de sostenibilidad. Etnobiología, 10(2), 12-28.

Comisión Nacional Forestal (CONAFOR). (2010). Estudio regional forestal (UMAFOR 0701). Recuperado de http://www.conafor.gob.mx:8080/documentos/ docs/9/3810Memoria\%20del\%20Estudio\%20Regional\%20Forestal\%200701.pdf

Comisión para el Conocimiento y Uso de la Biodiversidad (CONABIO). (2013). La biodiversidad en Chiapas: estudio de estado. Tuxtla Gutiérrez, Chiapas: Gobierno del Estado de Chiapas. Recuperado de http: / /www. biodiversidad.gob. $\mathrm{mx} / \mathrm{region} / \mathrm{EEB} / \mathrm{pdf} / \mathrm{chiapas}$ _vol1_baja.pdf

Consejo Nacional de Evaluación de la Política de Desarrollo Social (CONEVAL). (2016). Estadísticas de pobreza en Chiapas. Recuperado de https://www.coneval.org.mx/coordinacion/entidades/Chiapas/Paginas/principal.aspx

Constitución Política del Estado Libre y Soberano de Chiapas. (6 de septiembre de 2017). Periódico Oficial.

Constitución Política de los Estados Unidos Mexicanos. (9 de agosto de 2019). Diario Oficial de la Federación, Ciudad de México, 5 febrero de 1917. Última reforma en DOF (9 de agosto de 2019).

Contreras Hernández, J. (2005). La modernidad alimentaria. Entre la sobreabundancia y la inseguridad. Revista Internacional de Sociología, 63(40), 109132. doi: 10.3989/ris.2005.i40.191

Cuero, J. M. (2011). Desagrarización del campo chiapaneco. Encrucijadas: Revista Crítica de Ciencias Sociales, 1, 106-128. Recuperado de http://encrucijadas.org/index.php/ojs/article/view/117/111

Delgado, O., y Borges, P. A. (1965). Reformas agrarias en la América Latina. Mexico: Fondo de Cultura Económica.

Domínguez, D. (2015). La soberanía alimentaria como enfoque crítico y orientación alternativa del sistema agroalimentario global. Revista Pensamiento Americano, 8(15), 146-175. Recuperado de https://ri.conicet.gov.ar/bitstream/handle/11336/55264/CONICET_Digital_Nro.756ac4d8-cbfb-4ffc-86d17b69871f0849_A.pdf?sequence=2\&isAllowed=y

Durán, F. E. (2013). La producción social de los hábitos alimenticios. Una aproximación desde la sociología del consumo. Revista de Ciencias Sociales, 19(4), 683-693.

El Colegio de la Frontera Sur (ECOSUR). (2005). Programa estatal de reordenamiento territorial (PEOT) 2005. Recuperado de http://www.ecosur.mx/ sitios/catalogo_pub/.../programa-estatal-de-ordenamiento-territorial

Entrevista, trabajo de campo. (2018). Productor primario de mango, Tapachula, Chiapas.

Escalante, R., Catalán, H., Galindo, L. M., y Reyes, O. (2007). Desagrarización en México: tendencias actuales y retos hacia el futuro. Cuadernos de Desarrollo Rural, 4(59), 29-29. 
Escalante, S. R. y Talavera, F. D. (1998). La política macroeconómica en el sector agrícola. En F. T. Torres (coord.), El sector agropecuario mexicano después del colapso económico (pp. 73-100). México: Plaza y Valdés.

Fletes Ocón, H. B., Guzmán, G. O., y Ocampo, G. V. (2016). Reestructuración de la agricultura e inseguridad alimentaria. Las iniciativas y retos de los pequeños productores en Chiapas. EntreDiversidades. Revista de Ciencias Sociales y Humanidades, (7), 112-135.

Fletes Ocón, H. B., Rangel, F., Oliva Velas, A., y Ocampo Guzmán, G. (2013). Pequeños productores, reestructuración y expansión de la palma africana en Chiapas. región y sociedad, 25(57), 203-239. doi: 10.22198/rys.2013.57.a116

Flores, P., Vázquez, O. J., y Quintero Soto, M. L. (2012). ¿Soberanía, seguridad, autosuficiencia o crisis alimentaria? Caso de México y la región este de África. Problema básico en salud y calidad de vida. Rev. Dig. Universitaria, 13(8), 2-19.

Flores, A. G. (2017). Plantas útiles de los huertos frutícolas tradicionales de Coatetelco, Morelos, México frente al potencial emplazamiento minero. Acta Agrícola y Pecuaria, 3(3), 87-97.

Flores, E. (1960). La significación de los cambios del uso de la tierra en el desarrollo económico de México. El Trimestre Económico, 27(105), 1-14.

Fundación Produce, A. C. (2011). Programa de Desarrollo de Capacidades, Innovación Tecnológica y Extensionismo Rural. Tuxtla Gutierrez: Gobierno del Estado de Chiapas.

Galicia, J. V. (2016). Cartografías del deseo en Chiapas: entre la conservación y el extractivismo (tesis de maestría). El Colegio de la Frontera Sur, San Cristobal de las Casas, Chiapas. Recuperada de http: / / ecosur. repositorioinstitucional.mx/jspui/handle/1017/283

García, F. J. C., Gutiérrez, C. J. G., Balderas, P. M. A., y Araujo, S. R. (2016). Aprovechamiento de huertos familiares en el altiplano central mexicano. $R e$ vista Mexicana de Agroecosistemas, 3(2), 149-162.

García Jiménez, J. A., y Morales de la Rosa, M. J. (2017). Manual para el aprovechamiento de mango niño (Mangifera indica L.) en Chahuites, Oaxaca (tesis de Licenciatura en Ciencia y Tecnología de Alimentos-UNICACH). Facultad de Ciencias de la Nutrición y Alimentos. México. Recuperada de https://repositorio.unicach.mx/bitstream/20.500.12114/1227/1/CTA\%20664.8046\%20 G37\%202017.pdf

Gómez-Trujillo, E. A., Martínez-Andrade, E., Rivas-García, J. A., y Villalobos-Maradiaga, E. M. (2016). La seguridad y soberanía alimentaria. Revista Iberoamericana de Bioeconomía y Cambio Climático, 2(1), 315-324.

Gómez, N. (2017). La contribución socio-productiva de la organización social a la soberanía alimentaria en la Trinidad, Chiapas. C. Vázquez y k. Nicola. (eds.), México rural ante los retos del siglo XXI. Seguridad alimentaria, t. III (pp. 1128). México: Asociación Mexicana de Estudios Rurales A. C.

Grammont, H. C., de. (2001). El campo mexicano a finales del siglo XX. Revista Mexicana de Sociología, 63(4), 81-108. doi: 10.2307/3541469

Grammont, H., de. (2003). The agricultural sector and rural development in Mexico: consequences of economic globalization. En K. J. Middlebrook y E. 
Zepeda (eds.), Confronting Development: Assessing Mexico's Economic and Social Policy Challenge (pp. 350-381). Stanford, California: Stanford Univesrity Press.

Guzmán, E., Gómez, R., Pohlan, A. J., Álvarez, J., Pat, J., y Geissen, V. (2008). La producción de papaya en Tabasco y los retos del desarrollo sustentable. El Cotidiano, 23(147), 99-106.

Herrera, M. A. (2009). El monocultivo industrial de la palma africana y sus efectos en la seguridad alimentaria en Colombia (productos agrícolas) 2001-2006 (tesis maestría). Universidad de la Salle. Facultad de Ciencias Económicas y Sociales, México. Recuperada de https://ciencia.lasalle.edu.co/cgi/viewcontent.cgi?article=1030\&context=economia

Herrera, T. F. (2008). Apuntes sobre las instituciones y los programas de desarrollo rural en México. Del estado benefactor al estado neoliberal. Estudios Sociales, 17 (33), 18-38.

Instituto Nacional de Estadística y Geografía (INEGI). (2010). Principales resultados del Censo de población y vivienda 2010. México. Recuperado de http:// internet.contenidos.inegi.org.mx/contenidos/Productos/prod_serv/contenidos/espanol/bvinegi/productos/censos/poblacion/2010/princi_result/ mex/15_principales_resultados_cpv2010.pdf

Instituto Nacional de Estadística y Geografía (INEGI). (2012). Censo de población. Recuperado de http://www.beta.inegi.org.mx/servicios/datosabiertos.html y http://cuentame.inegi.org.mx/monografias/informacion/chis/poblacion/

Juárez, I. (2013). Carica papaya: una planta con efecto terapéutico. Horizonte Sanitario, 12(2), 35-36.

Landívar, N., López, G. J., y Yela, M. M. (2013). La palma africana en la provincia de Los Ríos: negocio agro-empresarial, prebendas estatales y violaciones de derechos campesinos. Eutopía, Revista de Desarrollo Económico Territorial, 2, $101-111$.

Lara, S., y De Grammont, H. C. (1999). Agricultura flexible y empleo en América Latina: un debate con el postfordismo. Innovación tecnológica y reorganización productiva en el sector agroexportador. México: Instituto de Investigaciones Sociales-Universidad Nacional Autónoma de México y Plaza y Valdés.

Lira Cossío, L. (2003). Cuestión regional y local en América Latina. Serie Gestión Pública. Santiago de Chile: Comisión Económica para América Latina y el Caribe.

López S., P. (2014). Entre la reconversión productiva y la soberanía alimentaria. El caso de la producción de jatropha en Chiapas. El Cotidiano, 188(7), 69-79. Martínez Castillo, R. (2010). Soberanía agroalimentaria: características, obstáculos y perspectivas. Ciencia y sociedad, 35(4), 623-656. Recuperado de https: / / www. redalyc.org/articulo.oa?id=325/32532787005

Méndez, O., y Gordillo, G. (2013). Seguridad y soberanía alimentaria; documento para discusión. Roma, FAO. 45. Recuperado de www.fao.org/publications Monroy-Martínez, R., Ponce-Díaz, A., Colín-Bahena, H., Monroy-Ortiz, C., y García-Flores, A. (2017). Los huertos familiares tradicionales soporte de seguridad alimentaria en comunidades campesinas del Estado de Morelos, México. Ambiente y Sostenibilidad, (6), 33-43. doi:10.25100/ays.v0i0.4288 
Monroy, R., Colín, H., Cruells, M. G., Flores, A. G., y Ayala, I. (2016). La gestión comunitaria de la diversidad biológica en riesgo por el crecimiento urbano en el municipio de Yautepec, Morelos, México. Etnobiología, 14(3), 50-59.

Morales, H., Aguilar-Støen, M. C., y Castellanos-López, E. J. (2015). Migración y remesas: ¿están afectando la sustentabilidad de la agricultura y la soberanía alimentaria en Chiapas? LiminaR, 13(1), 29-40.

Nicholls, C. I., y Altieri, M. A. (2011). Modelos ecológicos y resilientes de producción agrícola para el siglo XXI. Agroecología, 6, 28-37.

Organización de las Naciones Unidas para la Agricultura y la Alimentación (FAO). (2003). El estado de la inseguridad alimentaria en el mundo. Roma: Food \& Agriculture Org.

Paliwal, R. L. (2001). El maíz en los trópicos: mejoramiento y producción. Roma: Food \& Agriculture Org.

Piña, P. A. (2014). Cultura y alimentación. Aspectos fundamentales para una visión comprensiva de la alimentación humana. An. Antrop, 48(1), 11-31.

Poth, C. (2016). Semillas transgénicas y modelo agroalimentario: un debate ¿científico? Estudios Rurales, 6(10), 1-11.

Quintana, D. R. (1995). El paradigma neoliberal y las reformas agrarias en México. Cuadernos Agrarios, 5(11/12), 13-26.

Rodarte, B. O. A. (2015). Contradicciones y límites de la política agroalimentaria en México. De la seguridad alimentaria a la Cruzada contra el hambre. Argumentos, 28(79), 241-263.

Román-Ruiz, S. I., y Hernández-Daumas, S. (2010). Seguridad alimentaria en el municipio de Oxchuc, Chiapas. Agricultura, sociedad y desarrollo, 7(1), 7179.

Romero, A. A. (2016). Capitalismo y luchas socio-territoriales en Chiapas, México. Revista GeoNordeste, (2), 77-93.

Rosset, P. M. (2008). Mirando hacia el futuro: la reforma agraria y la soberanía alimentaria. AREAS. Revista Internacional de Ciencias Sociales, (26), 167182.

Rosset, P. M. (2014). Soberanía alimentaria: reclamo mundial del movimiento campesino. ECOfrontera, 18(51), 8-11. Recuperado de https://ecosur.repositorioinstitucional.mx/jspui/bitstream/1017/863/1/0000022841_documento. pdf

Rosset, P. M., y Torres, M. E. M. (2016). Agroecología, territorio, recampesinización y movimientos sociales. Estudios Sociales. Revista de Alimentación Contemporánea y Desarrollo Regional, 25(47), 273-299.

Salazar, R. L. (2014). Caracterización de la política social en México: limitaciones y retos. Revista Eleuthera, 11, 54-81.

Saldaña, A. G. , y Sánchez, A. P. (2010). Desarrollo local: configuraciones teóricas y sociales. En A. Pérez Sánchez (coord.), Desarrollo local: reflexiones teóricas y revisiones empíricas, p. 11-30. Tlaxcala: El Colegio de Tlaxcala.

Sánchez, J. G. A. , y Martínez, E. G. (2016). Contrastes de la agricultura familiar: el caso Benito Juárez, La Concordia, Chiapas. Revista de Geografía Agrícola, 56(2), 7-13. 
Sasson, A. (2000). La contribución de las biotecnologías a la alimentación. Biotecnología Aplicada, 17(1), 2-6.

Secretaría de Agricultura, Ganadería, Desarrollo Rural, Pesca y Alimentación (SAGARPA). (2018). Atlas agroalimentario 2012-2018, primera edición. MéxiCo: Servicio de Información Agroalimentaria y Pesquera.

Secretaría de Hacienda. (2011). Programa del Instituto de Reconversión Productiva y Bioenergéticos 2007-2012. México: Periódico Oficial, 330. Recuperado de http://www.haciendachiapas.gob.mx/planeacion/Informacion/Programacion_Sectorial/Programas_Institucionales/pdfs/77PROG_INST_IRPBIO.pdf

Servicio de Información Agroalimentaria y Pesquera (SIAP), y Secretaría de Agricultura, Ganadería, Desarrollo Rural, Pesca y Alimentación (SAGARPA). (2016). Acciones y programas en la producción agrícola. Recuperado de https://www.gob.mx/siap/acciones-y-programas/produccion-agricola-33119

Servicio de Información Agroalimentaria y Pesquera (SIAP), y Secretaría de Agricultura, Ganadería, Desarrollo Rural, Pesca y Alimentación (SAGARPA). (Varios años). Estadísticas de Producción Agrícola 2017, 2016, 2015, 2010, 2005, 2003, 2000, 1999, 1997 http://infosiap.siap.gob.mx/gobmx/datosAbiertos. php

Silva, J. G., da. (2017). Boom agrícola y persistencia de la pobreza rural en América Latina. Algunas reflexiones. Revista Austral de Ciencias Sociales, 18, 5-20. doi: 10.4206/rev.austral.cienc.soc.2010.n18-01

Solís, D. V. (2015). Transformaciones socioeconómicas y neo-extractivismo en Chiapas. Argumentos, 28(79), 191-213.

Valencia Sandoval, K., Duana Ávila, D., y Hernández Gracia, T. J. (2017). Estudio del mercado de papaya mexicana: un análisis de su competitividad (20012015). Suma de Negocios, 8(18), 131-139. doi: 10.1016/j.sumneg.2017.10.002

Valero P. J., Cortina V. H. S., y Vela C. M. P. (2011). El proyecto de biocombustibles en Chiapas: experiencias de los productores de piñón (Jatropha curcas) en el marco de la crisis rural. Estudios Sociales, 19(38),119-144.

Vallejo C. F. A. (2002). Mejoramiento genético de plantas. Cali: Universidad Nacional de Colombia.

Velasco, G. M. (1999). Globalización y subdesarrollo local: diferenciación social y migración en Chiapas. Papeles de Población, 5(22), 141-160.

Vidal, H. A. P., Ocón, H. B. F., y Cabañas, A. A. G. (2019). Agronegocios y campesinos maiceros en la Frailesca: vulnerabilidad y resistencias. Eutopía, Revista de Desarrollo Económico Territorial, 15, 11-31.

Villafuerte-Solís, D. (2010). Chiapas. La independencia y revolución que llegaron tarde. Economía y Sociedad, 14(25), 15-32.

Villafuerte-Solís, D. (2015). Crisis rural, pobreza y hambre en Chiapas. LiminaR, 13(1), 13-28.

Villafuerte-Solís, D., y García, A. M. D. C. (2014). Tres ciclos migratorios en Chiapas: interno, regional e internacional. Migración y Desarrollo, 12(22), 3-37.

Viñas, J. M. S. (2013). La volatidad de los mercados agrarios y la crisis alimentaria mundial. Cuadernos de Estrategia, (161), 157-187. 
Warman, A. (1980). Ensayos sobre el campesinado en México. México: Nueva Imagen.

Warman, A. (2001). El campo mexicano en el siglo XX. México: Fondo de Cultura Económica. 